6 Silver JR (1974) The prophylactic use of anti-coagulants in the prevention of pulmonary emboli in 100 consecutive spinal injury patients. Paraplegia 12: 188-196.

7 Walsh JJ, Tribe LR (1965) Phlebothrombosis and pulmonary embolism in Paraplegia 3: 209-213.

8 Damanski M (1961) Heterotopic ossification in paraplegia: a clinical study. J Bone Joint Surg 43B: 286-299.

9 Silver JR (1969) Heterotopic ossification: a clinical study of its possible relationship to trauma. Paraplegia 7: $220-230$.

10 Daud O, Sett P, Burr RG, Silver JR (1993) The relationship of heterotopic ossification to passive movements in paraplegic patients. Disabil Rehabil 15: 000-000.

\title{
Reply from Drs Colachis and Clinchot
}

Dr Silver suggests that following acute traumatic spinal cord injury (SCI), delayed admission to a spinal center results in an increased incidence of both deep venous thrombosis (DVT) and heterotopic ossification (HO), and that the delay in admission may account for the association observed in our study. We noted a statistically significant association between DVT and HO in patients with acute traumatic SCI admitted to our rehabilitation unit. Although we do believe that extrinsic compression may have accounted for cases of coexistent DVT and HO in our study, we do not contend that extrinsic compression alone can explain the findings. Certainly other factors could influence the association between DVT and HO. Any delay in admission resulting in increased incidence of both complications could play an important role, as Dr Silver suggested.

Excluding four patients admitted to our unit over 120 days following injury, the average time from injury to admission was approximately 30 days for patients who developed DVT and/or HO during their acute or rehabilitation hospitalization. Sixteen of our 30 patients with DVT had this condition diagnosed either before or at the time of admission to our unit. Although the vast majority of patients admitted received some form of prophylaxis (either heparin or compression pumps) prior to the development of DVT during their acute hospitalization, earlier admission to our unit might have altered our DVT incidence. It is of interest to note that the Model Systems data $^{1}$ from over 2000 acute SCI admissions, revealed a DVT incidence of $16.3 \%$ and $13.3 \%$ for early (day 1) and delayed (day 2-60) admissions, respectively. Patients treated at our institution do not routinely receive prophylaxis against or screening for the presence of $\mathrm{HO}$.

The highly variable incidence of both DVT and HO reported in the literature necessitates a large, well designed, prospective study to clarify the association between DVT and HO observed in our retrospective study. Factors which alter the incidence of both conditions could undoubtedly influence this association; however, casual effects remain to be determined. We agree with $\mathrm{Dr}$ Silver regarding early admission to medical centers specializing in the acute and rehabilitation management of traumatic SCI.

\section{References}

1 Maynard FM. Weingarden S, editors. Secondary complications of spinnal cord injury (1990) In: Apple DF, Hudson LM Spinal Cord Injury: The Model. Proceedings of the National Consensus Conference on Catastrophic Illness and Injury, December 1989. The Georgia Regional Spinal Cord Injury Care System. Shepard Center for Treatment of Spinal Injuries Inc. Atlanta. GA: 57-65. 